\title{
Respuesta dieléctrica y bioquímica de un recubrimiento PLA-PGA-HAp-Quitosano-Colágeno sobre Ti6Al4V
}

\author{
Dielectric and biochemical response of a \\ PLA-PGA-HAp-Chitosan-Collagen coated on Ti6Al4V
Nerly Deyanira Montañez Supelano ${ }^{1} \quad$ Darío Yesid Peña Ballesteros ${ }^{1}$ Hugo Armando Estupiñán Durán²

Recibido 24 de julio de 2014, aceptado 9 de septiembre de 2015 Received: July 24, 2014 Accepted: September 9, 2015

\begin{abstract}
RESUMEN
Se evaluó la interacción de un biomaterial polimérico con medio de cultivo y células osteoblásticas, electrodepositado sobre Ti6Al4V. El compuesto está integrado por ácido poliláctico-ácido poliglicólicohidroxiapatita, modificado con quitosano y colágeno. Se calculó la permitividad relativa con los datos de impedancia plasmada en un espectro dieléctrico que permitió identificar las dispersiones alfa y beta, relacionadas con el intercambio iónico y la polarización de la membrana celular. La adhesión y proliferación celular se analizaron mediante microscopia de epifluorescencia, en donde fue posible observar al tercer día del cultivo celular el proceso de mitosis representado por la condensación del núcleo y la separación de los cromosomas. Se observó la morfología de la superficie del biomaterial mediante SEM (Scanning Electron Microscope) y AFM (Atomic Force Microscope) y se evaluó la actividad celular mediante la medición de fosfatasa alcalina. Finalmente se encontró la mejor superficie para la adhesión y crecimiento celular mediante análisis estadístico, que correspondió al recubrimiento con la mayor concentración de quitosano y colágeno.
\end{abstract}

Palabras clave: Poliláctico, poliglicólico, hidroxiapatita, quitosano, colágeno, células, técnicas electroquímicas.

\begin{abstract}
The interaction of a polymeric biomaterial with a culture medium and osteoblast cells, electrodeposited on Ti6Al4V was evaluated. The compound is integrated of polylactic acid-polyglycolic acid-hydroxyapatite, modified with collagen and chitosan. The relative permittivity data embodied in a dielectric impedance spectrum identified the alpha and beta dispersions related to the ion exchange and the polarization of the cell membrane was calculated. Adhesion and cell proliferation was analyzed by epifluorescence microscopy, where it was observed on the third day of the cell culture process represented by mitosis core condensation and separation of the chromosomes. The surface morphology by SEM (Scanning Electron Microscope) and AFM (Atomic Force Microscope) biomaterial was observed and cellular activity was assessed by measuring alkaline phosphatase. Finally the best surface for adhesion and cell growth was found by statistical analysis, which corresponded to the coating with the highest concentration of chitosan and collagen.
\end{abstract}

Keywords: Polylactic polyglycolic, hydroxyapatite, chitosan, collagen, cells, electrochemical techniques.

1 Universidad Industrial de Santander. Calle 9 Carrera 27 Ciudad Universitaria. Bucaramanga, Colombia.

E-mail: ing.nerly.montanez@hotmail.com; dypena@uis.edu.co

2 Universidad Nacional de Colombia. Sede Medellín, Calle 59A No 63-20. Medellín, Colombia. E-mail: haestupinand@unal.edu.co 


\section{INTRODUCCIÓN}

La búsqueda de materiales biocompatibles que pueden ser usados en dispositivos médicos para la reparación de defectos y enfermedades óseas ha aumentado recientemente. Estos materiales deben ser apropiados para que funcionen como soporte de las células regeneradoras de hueso para que puedan proliferar y diferenciarse.

El estudio de propiedades eléctricas en el tejido óseo nació con el descubrimiento del efecto piezoeléctrico en su estructura y de las implicaciones de dicho fenómeno en el proceso de consolidación y tratamiento de patologías óseas [1,2]. Algunos materiales biológicos como el colágeno y biopolímeros exhiben una orientación polar uniaxial de los dipolos en su estructura molecular y pueden ser considerados como bioeléctricos. El colágeno comprende el $90 \%$ de la matriz ósea y junto con la hidroxiapatita (HA), gobierna las propiedades biomecánicas y la integridad de este tejido.

La caracterización de la interfase creada entre la superficie del implante y las células llevan a entender los complejos procesos implicados en los mecanismos de interacción para desarrollar superficies que faciliten la incorporación del dispositivo médico. Esta caracterización de la interacción entre células y biomaterial se realiza generalmente mediante medios ópticos y microscópicos, o retirando el biomaterial implantado en un animal. Estudios electroquímicos para medios con células vivas son menos frecuentes y todavía escasos en la literatura.

En este trabajo, el biopolímero PLA-PGA se modifica con materiales como colágeno, hidroxiapatita y quitosano. Los objetivos fueron la mejora de la respuesta inmune de células osteoblásticas y el estudio de la interacción polímero-material biológico desde el punto de vista de la respuesta electroquímica de la superficie, como consecuencia de la adsorción de proteínas, adhesión y crecimiento celular. Se modeló el barrido de frecuencias en un circuito de impedancia electroquímica, en el que fue posible interpretar los fenómenos eléctricos ocurridos en la interfase PLA-PGA modificado-proteínas de adsorción-células osteoblásticas. Además las técnicas microscópicas sirvieron para identificar la presencia de las células, la proliferación y morfología en las superficies de estudio.

\section{METODOLOGÍA}

La metodología experimental se basó en la evaluación por medio de técnicas electroquímicas de recubrimientos de ácido poliláctico, ácido poliglicólico e hidroxiapatita, modificados con quitosano y colágeno, depositados sobre sustratos de Ti6Al4V durante varios tiempos de cultivo celular. Los resultados permitieron conocer la aplicación de las técnicas electroquímicas, espectroscopia de impedancia electroquímica y voltametría cíclica sobre materiales biológicos. A continuación se describen las diferentes etapas de la metodología.

Síntesis de ácido poliláctico PLA: Ácido poliláctico se obtuvo a partir de ácido L-láctico Carlo Erba al $98 \%$, actuando como catalizador cloruro de zinc en una concentración de $1,5 \%$ en peso. El procedimiento se llevó a cabo en un rotoevaporador, con control de temperatura y presión de vacío. Se le suministró al sistema entrada de gas nitrógeno para mantener la reacción a atmosfera inerte. El tiempo total de síntesis fue de 57 horas. Se llevó a cabo la deshidratación a una temperatura de $100{ }^{\circ} \mathrm{C}$ y presión atmosférica, seguido de la esterificación. Esta reacción se mantiene a $180{ }^{\circ} \mathrm{C}$, a presión de 133 mbar de 6 a 7 horas y entrada de $\mathrm{N}_{2}$, la etapa ulterior fue la oligomerización, la que se da a una temperatura de $180{ }^{\circ} \mathrm{C}$ a una presión de 33 mbar y entrada de $\mathrm{N}_{2}$. Se dio inicio a la policondensación con la introducción del catalizador a una presión de 13 mbar durante 5 horas [3].

Síntesis de ácido poliglicólico PGA: El PGA se obtuvo mediante policondensación a partir de ácido glicólico Carlo Erba al 70\%, con 0,5\% de $\mathrm{ZnCl}_{2}$ como catalizador, y un tiempo total de síntesis de 25 horas. Se llevó a cabo la reacción entre el ácido y el catalizador con agitación a una temperatura de $190{ }^{\circ} \mathrm{C}$ bajo vacío de 195 mbar por una hora. Se redujo la presión hasta 39 mbar manteniendo la temperatura y sin agitación durante 4 horas. Se aumentó la temperatura a $230{ }^{\circ} \mathrm{C}$ hasta que el polímero se fundió totalmente y finalmente se mantuvo en un crisol a $190{ }^{\circ} \mathrm{C}$ por 20 horas [4].

Síntesis de hidroxiapatita HAp: Se usó el método de precipitación acuosa para obtener los cristales de hidroxiapatita, utilizando Nitrato de Calcio tetrahidratado $\left(\mathrm{Ca}\left(\mathrm{NO}_{3}\right)_{2} .4 \mathrm{H}_{2} \mathrm{O}\right)$ y Fosfato de amonio dihidrogenado $\left(\left(\mathrm{NH}_{4}\right) \mathrm{H}_{2} \mathrm{PO}_{4}\right)$ en concentraciones $1 \mathrm{M}$ 
y $0,48 \mathrm{M}$, respectivamente, adicionados en relación molar de 1,67. Se basificaron las soluciones antes de mezclar utilizando hidróxido de amonio con agitación continua durante 3 horas para obtener un $\mathrm{pH}$ entre 9,8-10. Se dejó la solución en envejecimiento por 144 horas a temperatura ambiente y luego se secó a $60{ }^{\circ} \mathrm{C}$ por 24 horas. Por último se llevó a cabo la calcinación a $1050{ }^{\circ} \mathrm{C}$ durante 1 hora [5].

\section{Caracterización de PLA, PGA, HAp y colágeno:}

Los espectros infrarrojos se realizaron usando un espectrofotómetro infrarrojo de Transformada de Fourier (Shimadzu 8400, Japón) en un rango de 4000 a $500 \mathrm{~cm}^{-1}$, con 1,5 gramos de muestra pulverizada. Ambos polímeros pulverizados se mezclaron en seco en una proporción de $70 \%$ en peso de ácido poliláctico y $30 \%$ en peso de ácido poliglicólico [6], luego se fundió la mezcla a $200^{\circ} \mathrm{C}$ durante 20 minutos, teniendo en cuenta que las temperaturas de descomposición de ambos polímeros se encuentran por encima de $200{ }^{\circ} \mathrm{C}$ [7]. La hidroxiapatita se caracterizó cualitativa y cuantitativamente mediante difracción de rayos $\mathrm{X}$ en un difractómetro de polvo (BRUKER con geometría DaVinci, D8 Advance, Alemania), a un voltaje de $40 \mathrm{kV}$, corriente de 30 $\mathrm{mA}$, con un estándar interno de corindón - óxido de aluminio y muestreo de $0,02^{\circ}(2 \theta)$.

Electrodeposición: A una solución de PLA-PGA al $15 \% \mathrm{p} / \mathrm{v}$ con acetona se le agregó hidroxiapatita en una concentración de $10 \% \mathrm{p} / \mathrm{v}$. Una solución más se hizo con hidroxiapatita al $10 \%$ y quitosano al $3 \% \mathrm{p} / \mathrm{v}$. Se irradiaron con luz UV las fibrillas de colágeno (Bovine Achilles Tendon) (C9879 sigma, USA) durante 30 minutos. Luego se mezclaron con PBS (Phosphate Buffered Solution), se agitó la mezcla durante 30 minutos y se congeló a $-80^{\circ} \mathrm{C}$ en un cuarto frío. Este procedimiento proporciona un proceso controlado de hinchamiento uniforme de las fibras, lo que favorece la disolución parcial del colágeno.

El sustrato es Ti6Al4V, empleado en ortopedia por tener varias características favorables en el uso de implantes tales como la resistencia a la corrosión, biocompatibilidad y valores bajos de módulo de elasticidad (116 GPa) [8]. La electrodeposición fue hecha sobre discos de esta aleación empleando un electrodo de referencia de acero inoxidable. Los recubrimientos se dejaron secar bajo luz UV y se observaron por microscopia óptica (Figura 1).

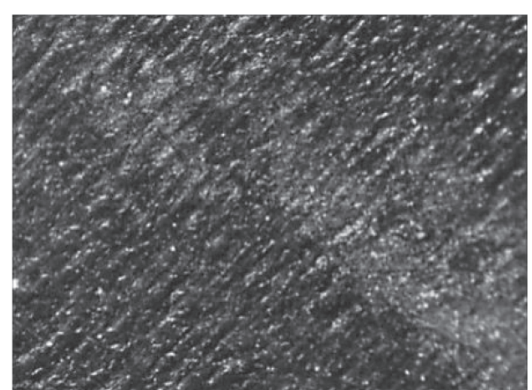

Figura 1. Microscopia óptica de los recubrimientos.

Cultivo celular: Se empleó la línea celular HOS (ATCC, American Type Culture Collection, EEUU), constituida por células osteoblásticas procedentes de un osteosarcoma humano. Se llevó a cabo el cultivo celular en frascos de cultivo Falcon ${ }^{\circledR}$ bajo condiciones estériles a $37{ }^{\circ} \mathrm{C} \pm 0,5{ }^{\circ} \mathrm{C}$ y $5 \%$ de $\mathrm{CO}_{2}$, en medio de cultivo RPMI 1640, con $1 \%$ de L-glutamina, $1 \%$ de antibióticos (Penicilina/ Estreptomicina) y suplementado con $10 \%$ de SBF (Suero Fetal Bovino), sin fenol rojo, pH de 7,2.

Ensayos electroquímicos: Se usó una celda electroquímica con dimensiones pequeñas y dos capilares Luggin, con un área de exposición de la muestra de $0,785 \mathrm{~cm}^{2}$. Se usó como electrodo auxiliar un electrodo de platino, como electrodo de referencia un electrodo de calomel saturado y como electrodo de trabajo el disco de Ti6Al4V recubierto de $1 \mathrm{~cm}$ de diámetro. Tanto el electrodo de referencia como el auxiliar, se introdujeron en los capilares Luggin ajustados en su extremo inferior con dos puntas vycor. Estos se llenaron con $\mathrm{KCl} 3 \mathrm{M}$ autoclaveado. La celda con la muestra se introdujo en una incubadora y todo el sistema se conectó a un potenciostato (Gamry ZRA 600, USA) en donde se hizo el seguimiento electroquímico del comportamiento de las células HOS sobre el biomaterial por medio de las técnicas electroquímicas: Potencial de corrosión, espectroscopia de impedancia electroquímica y voltametría cíclica, a tiempos de cultivo de 1,3 y 5 días.

Los parámetros empleados para las mediciones de impedancia fueron: barrido de frecuencia de $1 \mathrm{MHz}$ a $0,01 \mathrm{~Hz}$, siete puntos por década, voltaje AC $10 \mathrm{mV}$, voltaje DC $0 \mathrm{mV}$. Antes de realizar las mediciones de espectroscopia de impedancia electroquímica se registró el potencial de corrosión durante 300 segundos hasta que el potencial estabilizó. Para las mediciones de voltametría cíclica se realizó 
un barrido de potencial de -1 a $1 \mathrm{~V}$ respecto del electrodo de referencia, velocidad de barrido 50 $\mathrm{mV} / \mathrm{s}$, amplitud de $10 \mathrm{mV}$, corriente máxima de $0,1 \mathrm{~mA}$ y 4 ciclos.

\section{Medición de fosfatasa alcalina y proliferación} celular por microscopia de epifluorescencia: Para observar la actividad de los osteoblastos se realizó la medición de fosfatasa alcalina con una concentración celular de $30000 \mathrm{cel} / \mathrm{mL}$. Se tomaron los discos recubiertos e irradiados con luz UV y se depositaron en una placa de cultivo de 24 pozos. Se tomaron las células en fase exponencial, posteriormente se colocaron en cada pozo y se dejaron a $37^{\circ} \mathrm{C}$ en una atmosfera de $5 \%$ de $\mathrm{CO}_{2}$. Las mediciones se realizaron a 72 y $120 \mathrm{~h}$. A cada tiempo de medición se tomó tanto el sobrenadante como el lisado de las células adheridas. Para la lisis se adicionó tripsina-EDTA por $10 \mathrm{~min}$, se resuspendió en PBS, se centrifugó a 2500 rpm por 5 min y se resuspendió el pellet celular en Triton X-100 5\%.

La línea celular HOS (ATCC) fue cultivada en medio de cultivo RPMI-1640 suplementado con $10 \%$ de Suero Bovino Fetal (SBF). Se retiró la probeta del pozo, se lavó y se fijó por $10 \mathrm{~min}$. Se coloreó con Hoechst $1 \mu \mathrm{g} / \mathrm{mL}$ por $10 \mathrm{~min}$ en oscuridad. La lectura se realizó en un microscopio de epifluorescencia (Nikon Eclipse E400, Japón), utilizando el filtro UV2A.

Microscopia de fuerza atómica (AFM): Se prepararon los discos para microscopia fijando las células con $50 \mu \mathrm{l}$ de metanol, se dejaron secar a $-4{ }^{\circ} \mathrm{C}$ durante 48 horas. Seguido, se lavaron con PBS y se repitió el procedimiento anterior para la postfijación. Las muestras para AFM (Auto Probe CP, SCIENTIFIC INSTRUMENTS, USA) se llevaron hasta el punto de postfijación. Las imágenes digitales se analizaron mediante un software WSxM (Scanning Probe Microscopy, USA).

\section{RESULTADOS Y DISCUSIÓN}

Se muestra el espectro FTIR para el ácido poliláctico sintetizado y el ácido poliglicólico sintetizado (ver Figuras 2 y 3 ).

En el espectro infrarrojo de la Figura 2 se observan los picos característicos de los grupos funcionales

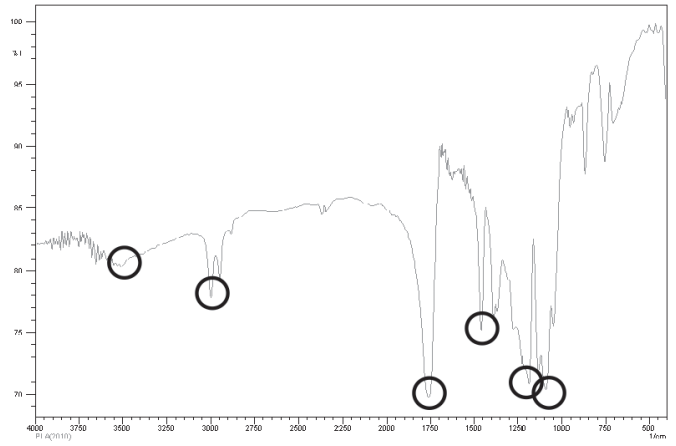

Figura 2. Espectro Infrarrojo PLA.

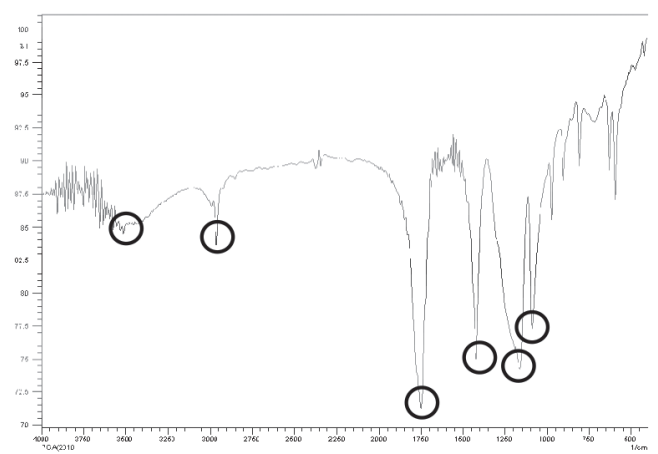

Figura 3. Espectro Infrarrojo PGA.

de la estructura del ácido poliláctico. Los grupos funcionales $\mathrm{O}-\mathrm{H}, \mathrm{C}-\mathrm{H}, \mathrm{C}=\mathrm{O}, \mathrm{CH}_{3}, \mathrm{C}-\mathrm{C}, \mathrm{C}-\mathrm{O}$, se ubican en los números de onda de 3510, 3000, $1756,1450,1190$ y $1086 \mathrm{~cm}^{-1}$ respectivamente [9].

Los grupos funcionales $\mathrm{O}-\mathrm{H}, \mathrm{C}-\mathrm{H}, \mathrm{C}=\mathrm{O}, \mathrm{CH}_{3}, \mathrm{C}-\mathrm{C}$, C-O se ubican en las longitudes de onda: 3500 , 2950, 1750, 1425, 1160 y 1090, respectivamente (ver Figura 3). Según Pandey y sus colaboradores [10], para el PGA las bandas típicas se encuentran alrededor de $2960 \mathrm{~cm}^{-1}(-\mathrm{CH}), 1430 \mathrm{~cm}^{-1}\left(-\mathrm{CH}_{3}\right)$, $1102 \mathrm{~cm}^{-1}$ (-C-O) y una señal de banda fuerte alrededor de $1727 \mathrm{~cm}^{-1}$ es característica del grupo $[\mathrm{C}=\mathrm{O}]$, que para el espectro infrarrojo del ácido poliglicólico obtenido se ubica en $1750 \mathrm{~cm}^{-1}$.

El análisis cualitativo de las fases presentes en la hidroxiapatita se realizó mediante comparación del perfil observado con los perfiles de difracción reportados en la base de datos PDF-2 del International Centre for Diffraction Data (Número de tarjeta PDF-2 para hidroxiapatita 010-74-7080, y para whitlockita 000-55-0898), referencia ICDD) (Figura 4). 
La caracterización de la HAp por difracción de rayos $\mathrm{X}$ mostró la presencia de fases cristalinas de hidroxiapatita y fosfato tricálcico (whitlockita). Este último se presenta en menor proporción, reportándose como un precursor de la HAp [11] (Tabla 1). El análisis cuantitativo de las fases encontradas se realizó mediante el refinamiento por el método Rietveld del perfil observado habiéndole agregado a la muestra una cantidad conocida estándar interno (Aluminum oxide, -100 mesh, $99 \%$, Corundum, $\alpha$-phase. Aldrich No. 23,474-5) correspondiente al $20 \%$.

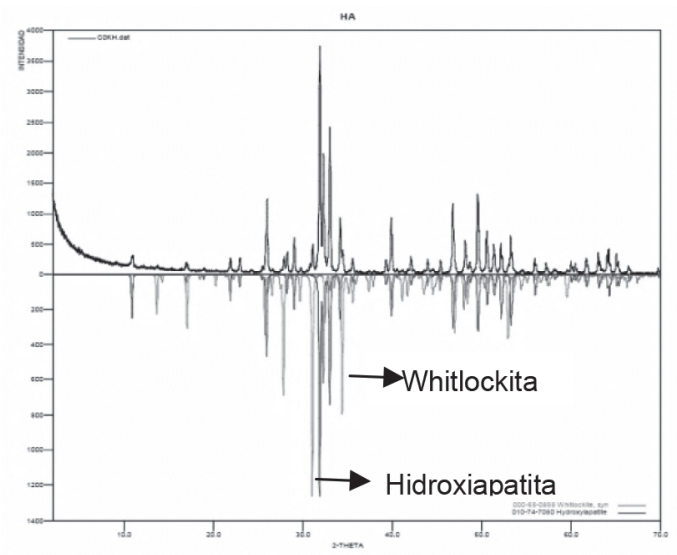

Figura 4. Diagrama de difracción de rayos X de la hidroxiapatita obtenida .

Tabla 1. Fases de la hidroxiapatita sintetizada.

\begin{tabular}{|c|c|c|c|}
\hline \multicolumn{2}{|c|}{ Fase } & Nombre & $\begin{array}{c}\text { Cuanti- } \\
\text { tativo }\end{array}$ \\
\hline \multirow{2}{*}{$\begin{array}{c}\text { CRISTA- } \\
\text { LINOS }\end{array}$} & $\begin{array}{c}\mathrm{Ca}_{3}\left(\mathrm{PO}_{4}\right)_{2} \\
(\mathrm{OH})\end{array}$ & Hidroxiapatita & $\begin{array}{c}84.9 \% \\
(\mathrm{DE}=0,5)\end{array}$ \\
\cline { 2 - 4 } & $\mathrm{Ca}_{3}\left(\mathrm{PO}_{4}\right)_{2}$ & Whitlockita & $\begin{array}{c}15.1 \% \\
(\mathrm{DE}=0,4)\end{array}$ \\
\hline
\end{tabular}

La morfología superficial de los recubrimientos presentó cambios con la adición de quitosano, el que hace la superficie más uniforme. Superficies más irregulares se presentaron para los recubrimientos que tienen colágeno y no tienen quitosano (ver Figura 5).

Los recubrimientos sin quitosano evidenciaron desprendimiento luego del tercer día, lo que no sucedió cuando el recubrimiento contenía quitosano. El quitosano hace que el recubrimiento sea más adherente al sustrato. Los grupos amino del quitosano interaccionan con los grupos carboxilo del PLAPGA y con fosfatos de calcio, creando un efecto positivo para la inmovilización de biomoléculas,

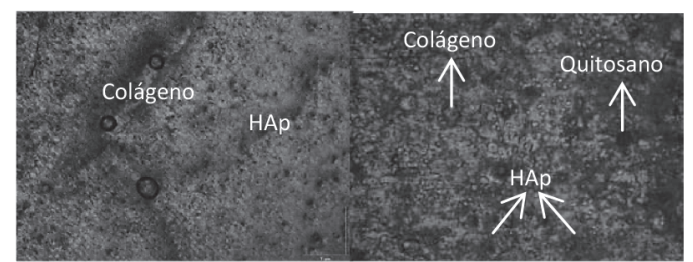

Figura 5. Microscopia óptica del recubrimiento a) PLA-PGA-HAp-Colágeno, 20X. b) PLA-PGA-HAp-Colágeno-Quitosano, 20X.

obteniéndose superficies más estables para la adhesión y proliferación celular [12-13].

Además las células se adhieren a los materiales por medio de las proteínas de adhesión como las integrinas (familia de glicoproteínas) y el colágeno. Estas generan diversos tipos de proteínas, ácidos orgánicos y aminoácidos. Un espacio se forma entre las células y el material debido a que la membrana de la célula está rodeada por proteínas de adhesión. De acuerdo con Hiromoto S. y colaboradores [14], una capa de adsorción de biomoléculas que contienen proteínas de las células se forma sobre la superficie del material y esta capa posiblemente previene la interacción de iones y moléculas del medio de cultivo por medio de la capa adhesiva de células, haciendo los potenciales de corrosión más nobles. Haciendo una relación entre la nobleza de los potenciales frente a la capa de adsorción de moléculas se observa que los potenciales correspondientes a células en todos los recubrimientos son más altos que en medio de cultivo, lo que indica la formación de la capa de proteínas de adhesión en presencia de células (ver Figura 6).

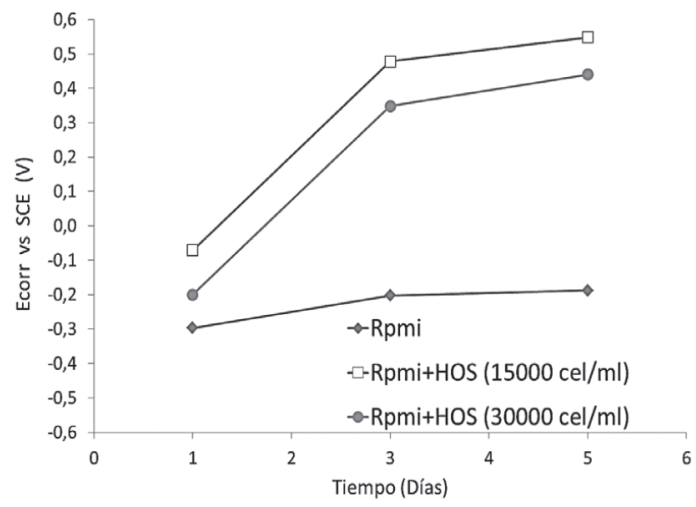

Figura 6. Evolución del potencial de corrosión. 
Los materiales biológicos presentan dos estructuras de compartimientos conductivos, espacios extra e intracelulares separados por membranas aislantes. La conducción de corriente eléctrica por los espacios extra e intracelulares es altamente dependiente de la frecuencia [15]. Los espectros de impedancia en los rangos de frecuencia reflejan las propiedades de las estructuras.
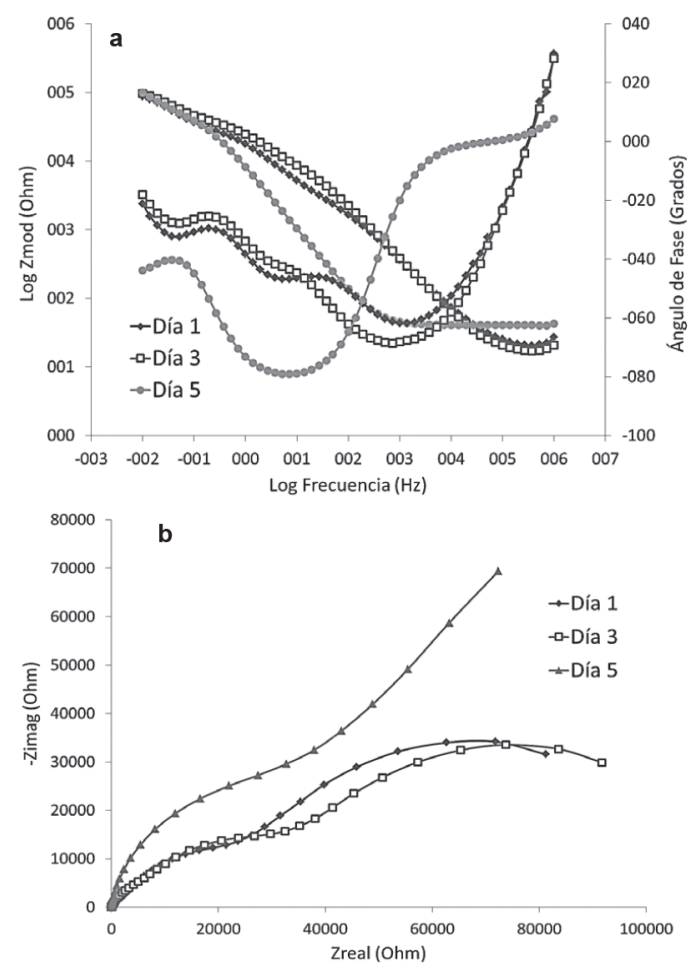

Figura 7. Espectros de impedancia obtenidos para el recubrimiento PLA-PGA-HApQuitosano(3\%)/Colágeno(0,05\%), concentración celular $30000 \mathrm{cel} / \mathrm{mL}$, a los diferentes tiempos de medición. (a) diagrama de Bode, (b) diagrama de Nyquist.

En la Figura 7, en la región de alta a media frecuencia, se encuentran los procesos que se llevan a cabo en la interfase medio de cultivo-células HOS. En la región de media a baja frecuencia se detectan los procesos de transferencia de carga y transferencia de masa que tienen lugar en la interfase proteínas o biomoléculas de adsorción-recubrimiento o al interior de los poros del recubrimiento [16]. Se observa la presencia de tres constantes de tiempo, atribuidas a los procesos en las interfases medio de cultivo Rpmi-células HOS, capa de biomoléculas de adsorción-recubrimiento y recubrimiento-sustrato de Ti6Al4V.

Los ángulos de fase para los recubrimientos con quitosano y con la mayor concentración de colágeno, en presencia de células, se mantienen entre $-70^{\circ}$ a $-80^{\circ}$ a medias frecuencias y aumentan a valores entre $-40^{\circ}$ a $-50^{\circ}$ a bajas frecuencias, asociados a procesos de control mixto en donde se llevan a cabo procesos de transferencia de carga y masa [16]. Esa transferencia de masa se define como la difusión de las proteínas de adsorción al interior de los poros del recubrimiento.

El incremento no lineal del módulo de impedancia en el barrido de frecuencias indica la presencia de más de una capacitancia, relacionadas con las constantes de tiempo. Estas capacitancias corresponden a la capacitancia de las células, la capa de adsorción de biomoléculas y el recubrimiento, mostrando las dos estructuras de compartimientos conductivos de los materiales biológicos.

Mediante circuito equivalente se ajustaron los datos obtenidos de impedancia para los ensayos con medio de cultivo y células (ver Figura 8).

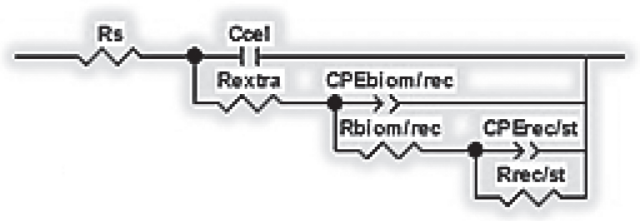

Figura 8. Circuitos equivalentes usados para el ajuste de los datos obtenidos de impedancia (a) Sin difusión y (b) con difusión.

En donde Rs es la resistencia a la solución, Ccel es la capacitancia de las células, Rextra es la resistencia asociada con la matriz extracelular excretada por las células en la interfase célulasbiomoléculas de adsorción, CPEbiom/rec es el elemento de fase constante debido a la interfase capa de biomoléculas adsorbidas-recubrimiento, Rbiom/rec es la resistencia asociada a la interfase biomoléculas adsorbidas-recubrimiento, CPErec/st es el elemento de fase constante de la interfase recubrimiento-sustrato, Rrec/st es la resistencia de dicha interfase. La representación del sistema biológico se muestra en Figura 9. 


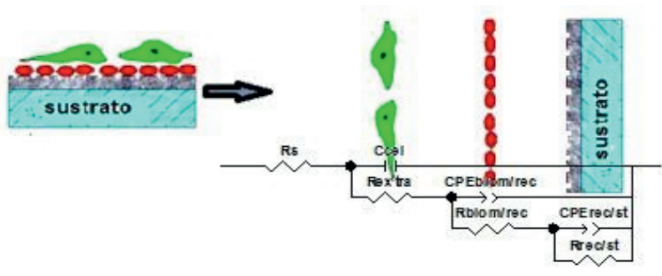

Figura 9. Representación del sistema biológico y su circuito equivalente.

A partir de la simulación de los circuitos equivalentes mediante el software Zview ${ }^{\circledR}$ se encontraron los valores de capacitancia y resistencia, que describen eléctricamente las diferentes interfases presentadas en los diferentes sistemas biológicos (ver Tabla 2).

Para determinar la permitividad de las mezclas poliméricas propuestas con medio de cultivo y células se empleó la ecuación 1 de permitividad compleja, donde la parte real corresponde a la permitividad relativa (ecuación 2) y la parte imaginaria (ecuación 3) es la pérdida dieléctrica [49], correspondiente a la pérdida disipativa asociada con el movimiento de cargas polarizables en fase con el campo eléctrico [17].

$$
\begin{gathered}
\tilde{\varepsilon}(\omega)=\varepsilon^{\prime}(\omega)-i \varepsilon^{\prime \prime}(\omega) \\
\varepsilon^{\prime}=\frac{C}{\left(1+\omega^{2} C^{2} R^{2}\right) * \varepsilon_{0}} \\
\varepsilon^{\prime \prime}=\frac{G}{\omega}+\frac{\omega C^{2} R}{\left(1+\omega^{2} C^{2} R^{2}\right) * \varepsilon_{0}}
\end{gathered}
$$

Donde $\omega=2 \pi f$ es la frecuencia angular, $C=1 / 2 \pi f Z^{\prime \prime}$ es la capacitancia, $R=Z^{\prime}$ es la resistencia, $G$ es la conductancia (parte real de la admitancia) y $\varepsilon_{0}=8,854 \times 10^{-12} F / m$ es la permitividad del vacío.

La parte real de la conductividad AC fue calculada usando la siguiente ecuación [18]:

$$
\sigma^{\prime}=G=\frac{Z^{\prime}}{Z^{\prime 2}+Z^{\prime 2}}
$$

En donde $Z^{\prime}$ es la impedancia real y $Z^{\prime \prime}$ es la impedancia imaginaria.

Una dispersión dieléctrica está asociada con los tejidos biológicos, en el que la permitividad relativa decrece con el aumento de la frecuencia [15]. Sin embargo, la corriente de desplazamiento es proporcional a la frecuencia del campo aplicado, y estos dos factores conducen a un comportamiento complicado de la frecuencia [15]. En la Figura 10 se muestra la permitividad relativa vs. $\log$ frecuencia para todos los recubrimientos a una concentración HOS de 30000 cel $/ \mathrm{mL}$ y 5 días de incubación celular.

De acuerdo con los valores encontrados de permitividad relativa y el barrido de frecuencias para el sistema células HOS-recubrimiento polimérico se identificaron dos dispersiones alfa y beta en los rangos de frecuencia de $0,01 \mathrm{~Hz}$ a $100 \mathrm{~Hz}$ asociado

\begin{tabular}{|c|c|c|c|c|c|c|c|c|c|c|c|c|}
\hline \multicolumn{3}{|c|}{$\begin{array}{c}\text { Ensayo } \\
\text { PLA-PGA/HAp+ }\end{array}$} & \multirow{2}{*}{ 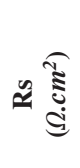 } & \multirow{2}{*}{ 己َّ } & \multirow{2}{*}{ 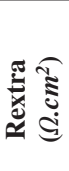 } & \multirow{2}{*}{ 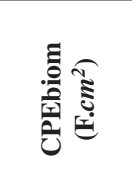 } & & \multirow{2}{*}{ 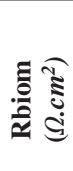 } & \multirow{2}{*}{ 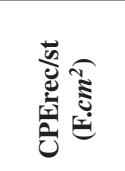 } & & \multirow{2}{*}{ 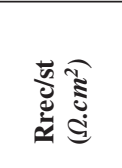 } & \multirow{2}{*}{ 离 } \\
\hline$\therefore$ & $\therefore \overline{0}$ & 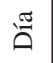 & & & & & & & & & & \\
\hline 0 & 0,05 & 1 & 15,07 & $5,297 \mathrm{E}-06$ & 100,8 & $1,687 \mathrm{E}-05$ & 0,776 & 4286 & $1,268 \mathrm{E}-05$ & 0,795 & 1,113E06 & 0,001636 \\
\hline 0 & 0,05 & 3 & 15,08 & 6,931E-06 & 197,4 & $1,377 \mathrm{E}-05$ & 0,748 & 6725 & $1,947 \mathrm{E}-06$ & 0,714 & $1,577 \mathrm{E} 07$ & 0,007842 \\
\hline 0 & 0,05 & 5 & 10,32 & 7,867E-06 & 104,2 & $1,333 \mathrm{E}-05$ & 0,775 & 9760 & $3,548 \mathrm{E}-06$ & 0,754 & $1,475 \mathrm{E} 07$ & 0,000292 \\
\hline 3 & 0,05 & 1 & 6,10 & $8,685 \mathrm{E}-06$ & 53,0 & $1,577 \mathrm{E}-05$ & 0,775 & 7754 & $5,472 \mathrm{E}-07$ & 1 & 1,895E06 & 0,000354 \\
\hline 3 & 0,05 & 3 & 4,51 & $8,652 \mathrm{E}-06$ & 55,0 & $1,228 \mathrm{E}-05$ & 0,819 & 7345 & $3,495 \mathrm{E}-06$ & 0,761 & 3,015E06 & 0,000627 \\
\hline 3 & 0,05 & 5 & 4,52 & $8,724 \mathrm{E}-06$ & 51,2 & $1,377 \mathrm{E}-05$ & 0,811 & 7712 & $2,475 \mathrm{E}-06$ & 0,845 & 2,712E05 & 0,001272 \\
\hline 0 & 0,075 & 1 & 9,24 & $1,065 \mathrm{E}-05$ & 70,7 & $1,379 \mathrm{E}-05$ & 0,828 & 4531 & $5,778 \mathrm{E}-06$ & 0,818 & 2,278E06 & 0,000365 \\
\hline 0 & 0,075 & 3 & 10,05 & 7,998E-06 & 52,2 & $1,453 \mathrm{E}-05$ & 0,764 & 4474 & $7,757 \mathrm{E}-07$ & 0,763 & $1,480 \mathrm{E} 06$ & 0,000148 \\
\hline 0 & 0,075 & 5 & 9,99 & $8,339 \mathrm{E}-06$ & 218 & $1,214 \mathrm{E}-05$ & 0,773 & 4520 & $2,918 \mathrm{E}-06$ & 0,676 & 3,082E06 & 0,001361 \\
\hline
\end{tabular}
con la interfase de los tejidos y de $100 \mathrm{~Hz}$ a $1 \mathrm{MHz}$ asociado con la polarización de la membrana celular,

Tabla 2. Valores de capacitancia y resistencia de las interfases de los sistemas biológicos. 


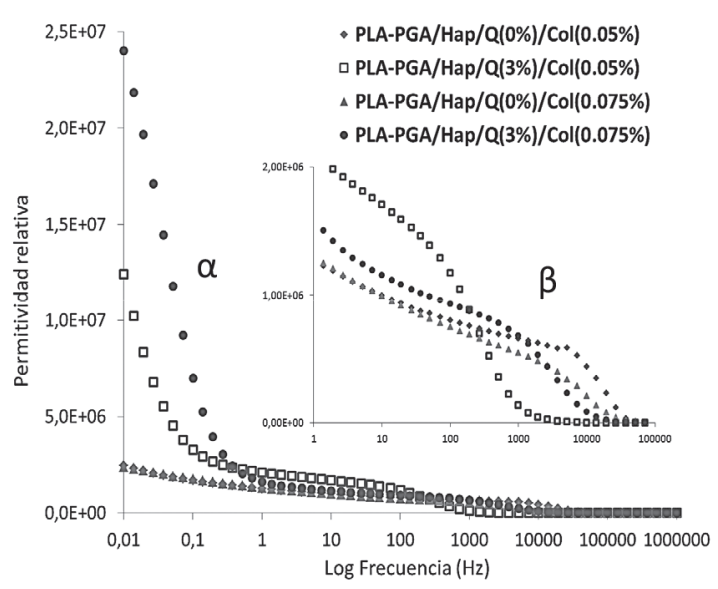

Figura 10. Variación de la permitividad con respecto a la frecuencia.

proteínas y otras macromoléculas orgánicas. La conductividad AC vs frecuencia para todos los recubrimientos a una concentración HOS de 30000 cel/mL se muestra en la Figura 11.

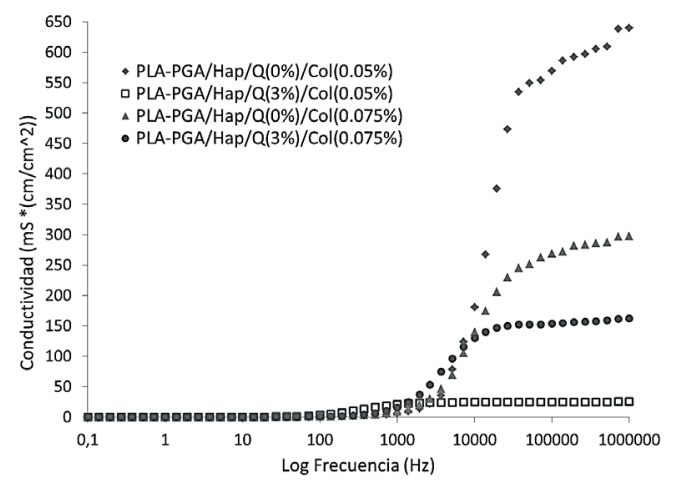

Figura 11. Variación de la conductividad con respecto a la frecuencia.

El alto valor de la permitividad a bajas frecuencias indican que los grupos de carga negativa como carboxilos ubicados en la superficie de las células forman una doble capa iónica con los iones positivos del medio, como se observa en la figura 10, a baja frecuencia la permitividad es mayor para el sistema biológico con el recubrimiento PLA-PGA/HAp/Q(3\%)/Col(0,075\%). A medida que la frecuencia aumenta, la permitividad disminuye, mostrando la tendencia para un tejido normal, permitiendo identificar la integridad de las membranas celulares adheridas a la superficie del recubrimiento. Los fenómenos de difusión iónica de los iones que envuelven la membrana celular (dispersión $\alpha$ ) juegan un papel importante en la determinación del comportamiento de los sistemas biológicos [19], así como la polarización de la membrana celular.

Por otra parte, el aumento en la conductividad para el recubrimiento PLA-PGA/HAp/Q $(0 \%) / \mathrm{Col}(0,05 \%)$, probablemente se deba a la rotura de membranas celulares por estrés (desprendimiento de la capa de colágeno). A alta frecuencia el flujo de corriente pasa tanto por el medio intra como extracelular [20-21], no se diferencia un tejido normal (células integras) a un tejido isquémico (algunas células muertas) (Höber 1912) [22], evidenciado además por el aumento de la impedancia a baja frecuencia.

La Figura 12 muestra un voltagrama de células HOS, adheridas sobre un sustrato de Ti6A14V, recubierto con polímero modificado, a las 72 horas de incubación celular, en donde se evidencian los picos de corriente catódica, presentados en la mayoría de los sistemas en estudio.

La altura de pico de corriente es de importancia en el análisis de los voltagramas, de acuerdo al estudio de Cobas y colaboradores, la altura de pico determina las fases de crecimiento en cultivos de células bacterianas [23]. En ese contexto, los voltagramas con las mismas condiciones de concentración celular y comparando los diferentes recubrimientos, los picos de corriente mayores indican un mejor crecimiento celular en un sistema dado.

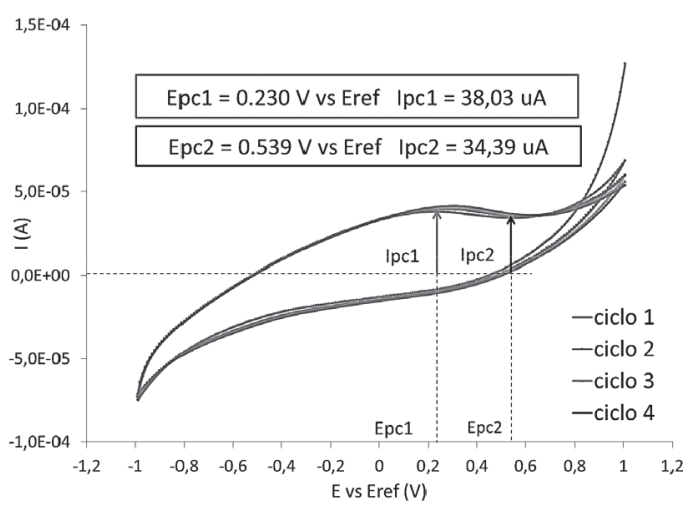

Figura 12. Voltagrama obtenido para el recubrimiento PLA- PGA/HAp/Q(3\%)/Col(0,075\%), concentración celular $30000 \mathrm{cel} / \mathrm{mL}$ a 72 horas de incubación celular. 
En la Figura 13 se muestran los voltagramas de los diferentes sistemas o recubrimientos correspondientes a 5 días de incubación, con una concentración HOS de $30000 \mathrm{cel} / \mathrm{mL}$, en donde se observa que el ancho de pico de corriente menor se presentó para el recubrimiento sin quitosano y con la menor concentración de colágeno.

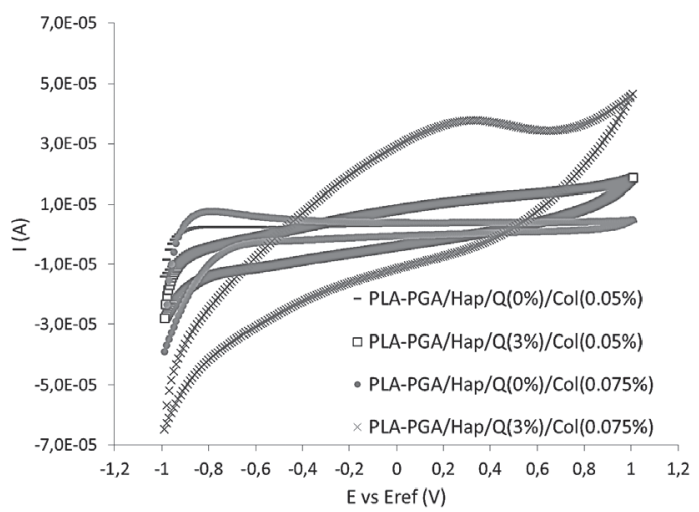

Figura 13. Voltagramas obtenidos para todos los recubrimientos, concentración celular de $30000 \mathrm{cel} / \mathrm{mL}$, tiempo de cultivo de 5 días.

Observando el valor más alto de pico de corriente (Figura 13), el recubrimiento con la mayor concentración de quitosano y colágeno tiene mayor valor de pico de corriente debido a la mayor actividad celular y adsorción de biomoléculas en la superficie del recubrimiento.

Se determinó la producción de fosfatasa alcalina tanto en los sobrenadantes como en los lisados celulares en diferentes tiempos de incubación de las células (72 y 120 h) sobre los discos de Ti6Al4V recubiertos. Se registraron los valores promedio y la correspondiente desviación estándar de las absorbancias para los diferentes sistemas con una concentración celular de $30000 \mathrm{cel} / \mathrm{mL}$ (ver Figuras 14 y 15).

La diferenciación de los osteoblastos se da a partir del tercer día [24], por lo que en ambos casos la absorbancia a 72 horas es menor que a 120 horas, incluso por debajo del control. Se evidenció la presencia de concentraciones de fosfatasa alcalina mayores en los sobrenadantes que en sus respectivos lisados celulares probablemente por el uso de EDTA, el que en ocasiones inhibe la acción de la fosfatasa alcalina, por lo que debe evitarse su empleo [25].

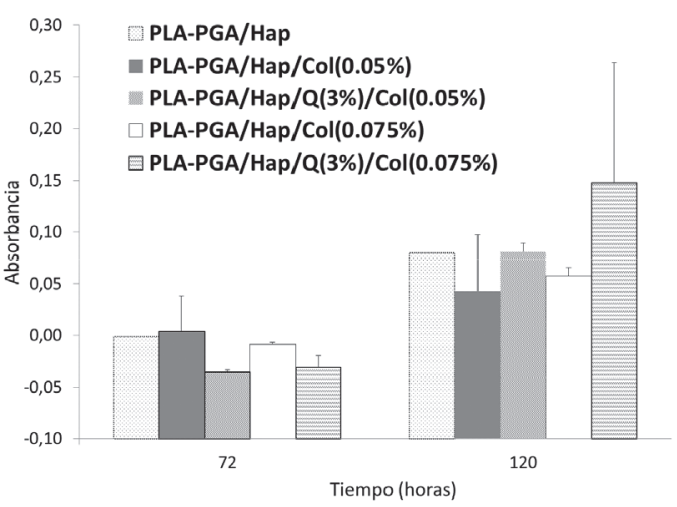

Figura 14. Producción de fosfatasa alcalina para los diferentes recubrimientos en los sobrenadantes.

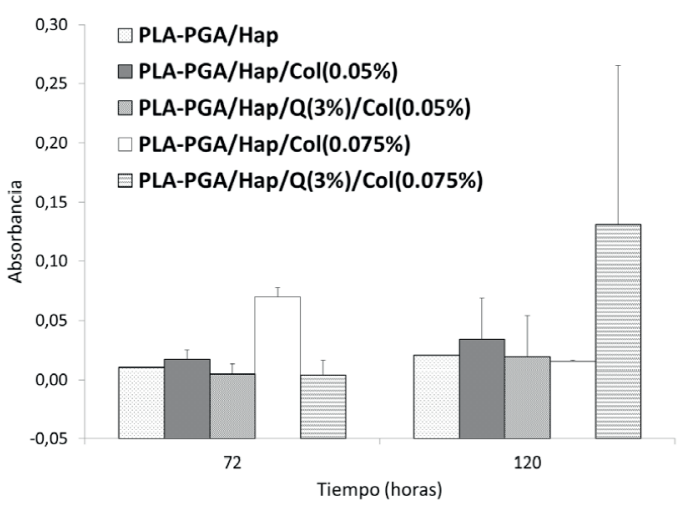

Figura 15. Producción de fosfatasa alcalina para los diferentes recubrimientos en los lisados celulares.

De acuerdo con Setzer y colaboradores [26], quienes realizaron estudios de producción de fosfatasa alcalina con coloración para células de tipo osteoblástico, al día 7 de cultivo, las células alcanzan confluencia, empiezan a diferenciar y solo algunas células son positivas para ALP. Con la iniciación de la producción de matriz (14 días) se puede observar muchas más células positivas para ALP. Las mediciones ALP para los polímeros modificados mostraron una gran variabilidad representado por desviaciones estándar amplias (como en el caso de PLA-PGA-HAP-Col (0,075\%), en los lisados celulares) probablemente debido a que a los cinco días de cultivo no es posible obtener datos lo suficientemente positivos para fosfatasa alcalina. El tiempo de ensayo se escogió a máximo 5 días debido a que simultáneamente se llevaban las pruebas electroquímicas. Sin embargo como se 
observa en las figuras anteriores el recubrimiento PLA-PGA/HAp/Q(3\%)/Col $(0,075 \%)$ indujo mayor diferenciación celular, apenas en su inicio, tanto en el sobrenadante como en el lisado de las células a 120 horas de análisis.

Los resultados de la microscopia de epifluorescencia evidencian que las células HOS son capaces de adherirse a la superficie de los recubrimientos. Se observa el aumento de la población celular comparando la hora 72 con la hora 120 de incubación, mostrando así que se lleva a cabo replicación de las células adheridas a la superficie del polímero. En todos los sistemas evaluados a las 72 horas, fue posible evidenciar la presencia de células en mitosis representado por la condensación del núcleo y la forma característica de la mitosis celular, primero duplicando el material genético (cromosomas), luego se separan los dos juegos de cromosomas, se divide el citoplasma y por último la célula duplica su tamaño. Esta etapa de la célula es visible en las fotos tomadas con el objetivo de 40X. El recubrimiento que presentó mayor confluencia y menor desprendimiento del recubrimiento es el de mayor concentración de colágeno y quitosano, concordando estos resultados con los observados en ALP. En las Figuras 16-18 se puede observar en el inciso a) la confluencia celular y en el inciso b) (círculos) la etapa de mitosis celular.

Ensayos SEM se llevaron a cabo en las muestras con 120 horas de incubación. Se observa en la Figura 19 que las células se mostraron extendidas sobre la superficie de los recubrimientos.

A $5000 \mathrm{X}$ se observa una estructura en forma de panal con macroporos $(5 \mu \mathrm{m})$ y microporos $(1$ $\mu \mathrm{m})$ (ver Figura 20), poros de importancia para el desarrollo celular y para la interacción con proteínas y enzimas [13].
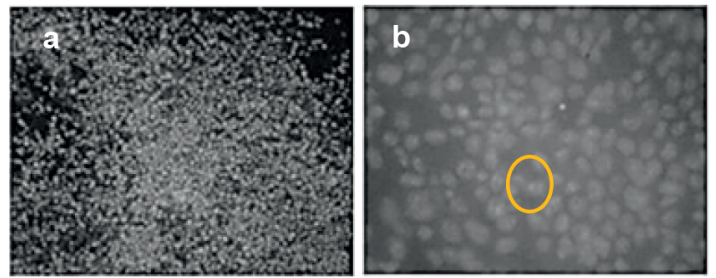

Figura 16. PLA-PGA/HAp/Q(3\%) $\mathrm{Col}(0.05 \%)$. (a) 10X, (b) 40X. 120 horas de incubación.
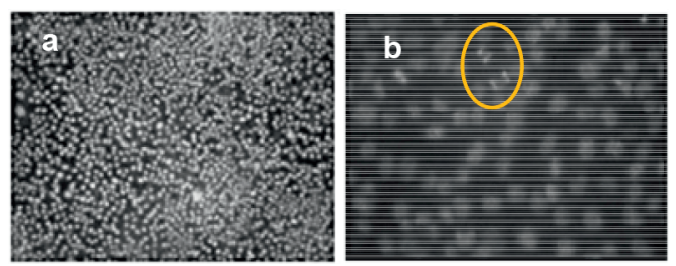

Figura 17.PLA-PGA/HAp/Q(3\%)Col(0.075\%). (a) 10X, (b) 40X. 72 horas de incubación.
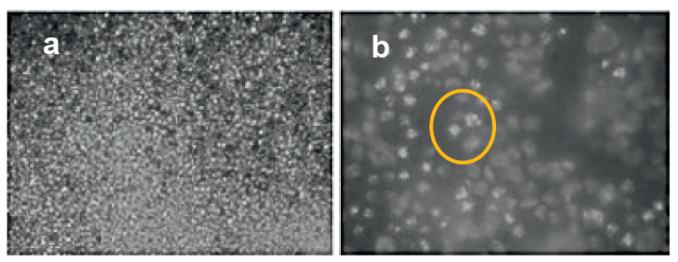

Figura 18.PLA-PGA/HAp/Q(3\%)Col(0,075\%). (a) 10X, (b) 40X. 120 horas de incubación.

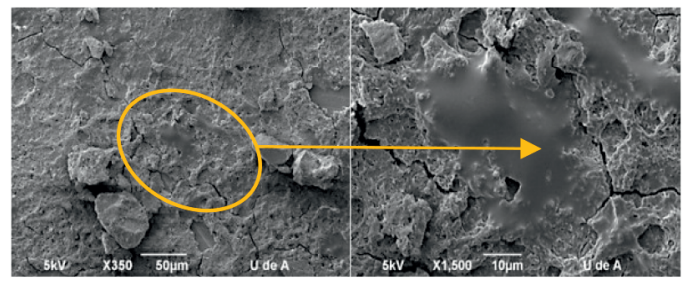

Figura 19. SEM para el recubrimiento PLAPGA/HAp/Q(3\%)/Col $(0,075 \%)$ y una concentración celular de $30000 \mathrm{cel} / \mathrm{mL}$. a) $350 \mathrm{X} \mathrm{y} \mathrm{b)} 1500 \mathrm{X}$.

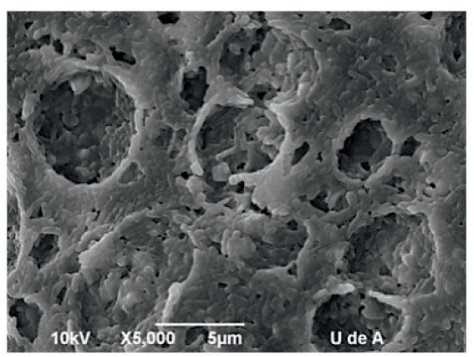

Figura 20. SEM para el recubrimiento PLA-PGA/ $\mathrm{HAp} / \mathrm{Q}(0 \%) / \operatorname{Col}(0,05 \%)$ a $5000 \mathrm{X}$.

Las micrografías resultantes de los análisis de AFM permitieron calcular la rugosidad de un área específica (ver Figura 21). Los recubrimientos con quitosano presentaron rugosidades promedio de $0,3293 \mu \mathrm{m}, \mathrm{y}$ los recubrimientos sin quitosano presentan un valor promedio de 0,6402 $\mu \mathrm{m}$. Respecto de los análisis electroquímicos, morfológicos y bioquímicos las células de tipo osteblástico HOS prefieren superficies porosas y de rugosidades relativamente pequeñas 
para la interacción de proteínas y biomoléculas, su fijación, proliferación y adherencia.

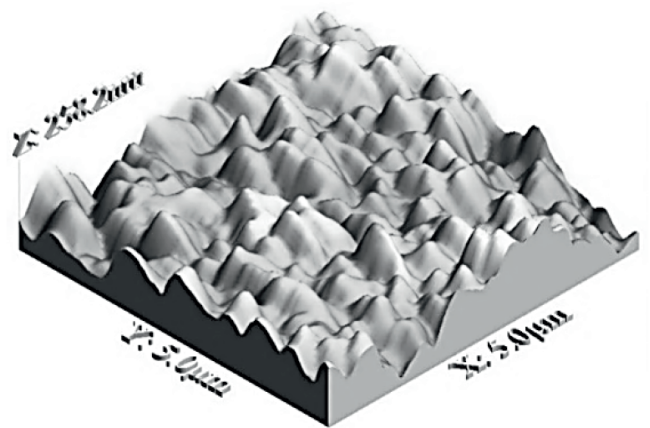

Figura 21. Micrografía por AFM en 3D, 30000 cel/ $\mathrm{mL}, 120$ h de incubación.

\section{CONCLUSIONES}

Se pudo identificar una interfase formada entre el recubrimiento de matriz polimérica y las células de tipo osteoblástico, asociada a una capa de adsorción de biomoléculas que contienen proteínas de las células, causando un aumento en los potenciales de corrosión a los tiempos de incubación celular, esa adsorción se presenta con la difusión de biomoléculas al interior de recubrimientos porosos que contienen quitosano y colágeno, los cuales proveen un ambiente estable de actividad proteínica.

El sistema biológico en estudio se ajustó a un modelo de circuito electroquímico, que describe eléctricamente las interfases presentadas, en donde la capacitancia de las células, identificada como Ccel, fue mayor para el recubrimiento PLA-PGA/ $\mathrm{HAp} / \mathrm{Q}(3 \%) / \mathrm{Col}(0,075 \%)$ y a los cinco días de incubación celular. De acuerdo con los espectros de permitividad relativa, se identificaron dos dispersiones, alfa y beta, en donde se evidenciaron los fenómenos de difusión iónica de los iones que envuelven la membrana (dispersión $\alpha$ ), asociada a mayor permitividad a baja frecuencia y la polarización de la membrana celular (dispersión $\beta$ ), los cuales permitieron identificar la integridad de las membranas celulares adheridas a la superficie de los recubrimientos.

Las características superficiales de un implante como morfología, rugosidad y porosidad, intervienen directamente en la adhesión y proliferación de los osteoblastos. En los recubrimientos fue posible mostrar el proceso de replicación de células mediante la mitosis celular a tres días de incubación en todas las superficies poliméricas en mayor proporción sobre el recubrimiento PLA-PGA / HAp/ Q(3\%)/ $\mathrm{Col}(0,075 \%)$, concordando con la medición de fosfatasa alcalina.

\section{AGRADECIMIENTOS}

Los autores extienden su agradecimiento a la Universidad Industrial de Santander - Grupo de Investigaciones en Corrosión (GIC)- Grupo Centro de Investigación Enfermedades Tropicales (CINTROP), a Colciencias y a la Universidad de Antioquia por la colaboración en los análisis SEM.

\section{REFERENCIAS}

[1] E. Moncada, A. Martínez, C. Pinedo y H. Cadavid. "Desarrollo e implementación de una técnica para la medición de impedancia en muestras de hueso húmedo bovino". Revista. Fac. Ing. Univ. Antioquia. Vol. 44, pp. 75-82. Junio 2008. ISSN: 0120-6230.

[2] Y. Zhang, L. Cheng, J. Zeng, K. Zhou and D. Zhang. "Aligned porous baruim titanate/hydroxyapatite composites with high piezoelectric coefficients for bone tissue engineering". Material Science and Engineering C. Vol. 39, pp. 143-149. February, 2014. ISSN: 0928-4931.

[3] D. Parada, H. Estupiñán, D. Peña, C. Vásquez y D. Laverde. "Obtención, electrodeposición y caracterización de un polímero bioabsorbible a partir de ácido L - láctico para aplicaciones biomédicas". Ingeniare. Revista chilena de ingeniería. Vol. $17 \mathrm{~N}^{\circ} 3$, pp. 365-374. Octubre 2009. ISSN: 0718-3305.

[4] K. Takahashi, I. Taniguchi, M. Miyamoto and Y. Kimura. "Melt/solid polycondensation of glycolic acid to obtain high-molecular-weight poly (glycolic acid)". Polymer. Vol. 41, pp. 8725-8728. 2000. ISSN: 0032-3861.

[5] C. García, C. Paucar y J. Gaviria. "Estudio de algunos parámetros que determinan la síntesis de hidroxiapatita por la ruta de precipitación". Revista Dyna. Vol. 73 No 148 , pp. 9-15. 2006. ISSN: 2346-2183.

[6] C. Motta e E. Duek. "Síntese, Caracterização e Degradação in vitro do poli (L-ácido láctico- 
co-ácido glicólico)". Revista Matéria. Vol. 11 No3, pp. 340-350. 2006. ISSN: 1517-7076.

[7] D. Garlotta. "A literature review of poly (lactic acid)". Journal of Polymers and the Environment. Vol. 9, Issue 2, pp. 63-84. Abril 2001. ISSN: 1572-8900.

[8] B. Ratner, A. Hoffman, F. Schoen and J. Lemons. "Biomaterials Science: An introduction to materials in medicine". Academic Press. Primera edición, pp. 41-50. San Diego, United States of America. ISBN: 0-12-582460-2. 1996.

[9] M. Perales, A. Torres y A. Flores. "Síntesis de polímeros: Ácido Poliláctico”. X Congreso Nacional de Microscopía-Morelia Michoacán. México. Mayo 2010.

[10] A. Pandey, G. Pandey and P. Aswath. "Synthesis of polylactic acid-polyglycolic acid blends using microwave radiation". Journal of the mechanical behavior of biomedical materials. Vol. 1, pp. 227-233. 2008. ISSN: 1751-6161.

[11] M. García y J. Reyes. "La hidroxiapatita, su importancia en los tejidos mineralizados y su aplicación biomédica”. Tip Revista Especializada en Ciencias QuímicoBiológicas, Vol. 9 No 2, pp. 90-95. 2006. ISSN: $1405-888 \mathrm{X}$.

[12] J. Wang, J. De Bóer and K. De Groot. "Preparation and Characterization of Electrodeposited Calcium Phosphate/Chitosan Coating on Ti6A14V Plates". Journal of Dental Research. Vol. 83, Issue 4, pp. 296301. Abril 2004. ISSN: 1544-0591.

[13] A. Martel, C. Martínez, J. Chacón, P. García and I. Olivas. "Synthesis and thermo-physical properties of chitosan /poly(dl-lactide-coglycolide) composites prepared by thermally induced phase separation". Carbohydrate Polymers. Vol. 81, Issue 4, pp.775-783. Julio 2010. ISSN: 0144-8617.

[14] S. Hiromoto, K. Noda and T. Hanawa. "Development of electrolytic cell with cellculture for metallic biomaterials". Corrosion Science. Vol. 44. Issue 5, pp. 955-965. Mayo 2002. ISSN: 0010-938X.

[15] D. Dean, T. Ramanathan, D. Machado and R. Sundararajan. "Electrical impedance spectroscopy study of biological tissues". Journal of Electrostatics. Vol. 66, Issue 3-4, pp. 165-177. Marzo 2008. ISSN: 0304-3886.
[16] A. García, L. Saldaña, C. Alonso, V. Barraco, M. Muñoz and M. Escudero. "In situ cell culture monitoring on a Ti-6Al-4V surface by electrochemical techniques". Acta Biomaterialia. Vol. $5 \mathrm{~N}^{\circ}$ 4, pp. 1374-1384. Mayo 2009. ISSN: 1742-7061.

[17] R. Pethig and D. Kell. "The passive electrical properties of biological systems: their significance in physiology, biophysics and biotechnology". Physics in Medicine Biology, Vol. 32, Issue 8, pp. 933-970. Agosto 1987. ISSN: 1361-6560.

[18] J. Gittings, C. Bowen, A. Dent, I. Turner, F. Baxter and J. Chaudhuri. "Electrical characterization of hydroxyapatite-based biocerámicos". Acta Biomaterialia. Vol. $5 \mathrm{~N}^{\circ} 2$, pp. 743-754. Febrero 2009. ISSN: 1742-7061.

[19] O. Casas. "Contribución a la obtención de imágenes paramétricas en tomografía de impedancia eléctrica para la caracterización de tejidos biológicos", pp. 5.1-5.78. 1998. Marzo 2011. URL: http://petrus.upc.es/ $\sim$ wwwdib/tesis/ Oscar/resumen.html

[20] R. Amit, I. Shur, D. Ramiz, R. Ragini, N. Fishelson, N. Croitoru, D. Benayahu and Y. Diamand. "Dielectric screening of early differentiation patterns in mesenchymal stem cells induced by steroid hormones". Bioelectrochemistry. Vol. 78, pp. 161-172. 2010. ISSN: 1567-5394.

[21] K. Heileman, J. Daoud and T. Maryam. "Dielectric spectroscopy as a viable biosensing tool for cell and tissue characterization and analysis". Biosensors and Bioelectronics. Vol. 49, pp. 348-359. 2013. ISSN: 0956-5663.

[22] A. Otero. "Aplicación de la dielectroforesis a la caracterización dieléctrica de células", pp. 10-30. Mayo 2008. Marzo 2011. URL: http://eprints.ucm.es/8150/1/T30510.pdf

[23] H. Cobas, S. Pavoni, A. Zayas, F. Rodríguez, E. Álvarez y R. Contreras. "Aplicación de la Voltametría en el Análisis del Crecimiento Microbiológico". VII Congreso de la Sociedad Cubana de Bioingeniería. La Habana, Cuba. Julio 2007.

[24] G. Colaianni, C. Cuscito, T. Mongelli, A. Oranger, G. Miori, G. Brunetti, S. Colucci, S. Cinti and M. Grano. "Irisin Enhances Osteoblast Differentiation In Vitro". International Journal of Endocrinology. Vol. 2014, pp. 2-5. 2014. URL: http://www. hindawi.com/ journals/ije/2014/902186/ 
[25] Wuhan De Sheng Technology Co., Ltd. "Dihidrato de ácido etilendiaminotetraacético dipotásico". Abril 2011. URL: http:// whdstech.com/es/ products/biochemicalreagents-and-blood-tests/item/90 -ethylenediaminetetraacetic-acid-dihydratedipotassium.html

[26] B. Setzer, M. Bächle, M. Metzger and R. Kohal. "The gene-expression and phenotypic response of hFOB 1.19 osteoblasts to surface-modified titanium and zirconia". Biomaterials, Vol. 30, Issue 6, pp. 979-990. Febrero 2009. ISSN: 0142-9612.

[27] P. Zhang, Z. Hong, T. Yu, X. Chen and X. Jing. "In vivo mineralization and osteogenesis of nanocomposite scaffold of poly (lactide-coglycolide) and hydroxyapatite surface-grafted with poly (L-lactide)". Biomaterials. Vol. $30 \mathrm{~N}^{\mathrm{o}}$ 1, pp. 58-70. Enero 2009. ISSN: 0142-9612. 